\title{
Simulación y optimización de un horno cerámico monocapa
}

\author{
J. UCHE, J. Ma MARÍN \\ Departamento de Ingeniería Mecánica.Universidad de Zaragoza. ESPAÑA.
}

\begin{abstract}
La modelización matemática de un horno cerámico monocapa de rodillos, desarrollando los complejos modos de transmisión de calor existentes, se ha realizado con la ayuda de un programa informático. Con el desarrollo de diferentes parámetros de diseño, así como diferentes pastas cerámicas y diferentes composiciones de gas pueden ser introducidas en dicho programa, para estudiar la correspondiente optimización paramétrica del horno. Los resultados globales de las simulaciones están en acuerdo con los datos de consumo específicos aportados por la industria azulejera.
\end{abstract}

Palabras clave: Cerámica, horno, rodillos, monocapa, simulación.

\section{Simulation and Optimisation of a Single-Deck Roller Kiln for Ceramic Products}

Mathematical model of a single-deck roller kiln, developing the complex heat transfer modes acting inside the kiln, is made using a computer program. Design parameters, as well as types of tiles and gas consumption can be introduced in the program, in order to study the parametric optimisation of the model. Global results of the simulations have a good agreement with the data obtained from the ceramic industries.

Keywords: Roller kiln, tiles, ceramic, optimisation, simulation.

\section{INTRODUCCIÓN}

La aparición de los hornos cerámicos monocapa hacia el año 1.985, ha comenzado a sustituir los tradicionales hornos túnel, de mayor capacidad de producción pero de ciclo mucho más largo que los hornos monocapa. El horno monocapa tiene un rendimiento energético más elevado que los hornos tradicionales debido a la disminución del ciclo de cocción conseguido mediante un diseño que aumenta considerablemente la velocidad de transmisión de calor, y ofreciendo a la vez una mayor calidad en la producción debido a la homogeneidad de temperaturas en el horno. Estos hornos se emplean con profusión en la cocción de esmalte (bicocción) ó en monococción y recientemente se están empezando a emplear en cocción de bizcocho. El desarrollo de esta técnica de fabricación presenta las ventajas de disminuir el consumo energético, aumentar la calidad de producción, flexibilizar el proceso productivo y facilitar la automatización de la producción. La implantación de esta técnica constituye una vía para reconvertir el mercado de hornos túnel de bizcocho transformados a gas y captar el mercado de hornos túnel a fuel-oil.

Otras de las ventajas de los hornos monoestrato se enumeran a continuación:

- Reducción de los costes de producción: mano de obra, energía eléctrica y consumo de gas, etc.

- Reducción de la inversión por una menor ocupación de espacio, alrededor del 70\% inferior al ciclo tradicional.

- Reducción del stock de material sometido al proceso de fabricación, debido al alto nivel de automatización del sistema.

- Mayor uniformidad en las características técnicas del bizcocho con mejor estirada del esmalte, textura y uniformidad de tonos.
- Posibilidad de controlar mejor el proceso productivo, con lo que se reduce el porcentaje de piezas rechazadas.

La cocción de la pasta cerámica es un proceso muy delicado que necesita una elevación gradual de temperaturas del proceso de cocción para evitar una rotura o dañado de las piezas. El enfriamiento de la pasta cerámica tras el proceso de cocción tampoco puede hacerse bruscamente, especialmente en algunos márgenes de temperatura, que luego se tendrán en consideración.

Si la pasta cocida no ha pasado por los valores de temperatura y tiempo adecuados para su composición específica, sus condiciones de fragilidad, resistencia mecánica y plasticidad no son óptimas, repercutiendo negativamente en la calidad del producto, y por lo tanto, a las ventas de la producción cerámica, el sector cerámico es suficientemente competitivo como para considerar necesario el ofrecer al cliente la mejor calidad posible de producto.

El objetivo de este artículo es desarrollar el modelado teórico de los mecanismos de transmisión de calor y de la combustión para reproducir informáticamente el comportamiento real de dicho horno. Además de estudiar la intercambiabilidad de los gases naturales en la producción del horno, el modelo permitirá estudiar la evolución de diferentes parámetros constituyentes del horno, de cara a la optimización energética del mismo. Entre estos parámetros cabe destacar entre otros, parámetros geométricos: número de módulos del mismo (el horno tiene estructura modular), dimensiones de cada módulo. Otros parámetros pueden ser el cambio de los rodillos del sistema de movimentación, cambios en la geometría del quemador; así como diferentes variables relacionadas con el proceso de cocción y enfriamiento: caudales de aire de combustión y enfriamiento, etc. 
Hasta el momento, no existe ningún modelo tan detallado y con tantas opciones a estudiar de este tipo de hornos, por lo que parece de mucha utilidad para los investigadores de los procesos productivos de la industria cerámica. Tras la validación del modelo en una posterior fase experimental, a partir de una contrastación de los datos obtenidos, podrá servir a los ceramistas como base de pruebas para verificación del comportamiento de las muestras ante distintos estados de funcionamiento del horno, según sus variables de entrada. El programa por lo tanto podrá simular el comportamiento de una pasta cualquiera en un horno real, con el consiguiente ahorro de tiempo y dinero para los industriales de la zona (no necesitarán un horno de una producción de miles de $\mathrm{m}^{2} /$ día infrautilizándolo en pruebas de una hornada en particular).

\section{DESCRIPCIÓN DEL MODELO}

El horno monoestrato podría describirse como un largo canal interior, en torno a los 80-100 metros de longitud, en cuyo centro tiene insertada una fila de rodillos de pequeño diámetro que atraviesan toda la anchura del canal. Dichos rodillos sirven de apoyo y movimentación a la pasta cerámica, colocada en toda la anchura posible y formando una sola capa (en eso se diferencia de los hornos túnel convencionales), que de esta forma atraviesa en un corto espacio de tiempo toda la longitud del horno; por lo tanto reducimos el ciclo de cocción y enfriamiento de la pasta cerámica. La altura del canal será por lo tanto muy escasa, la suficiente para que la atmósfera del canal pueda calentar las piezas cerámicas.

En la zona más cercana a la entrada de la pasta, nos encontramos con el flujo de gases de combustión de los quemadores situados posteriormente, por lo tanto en esta primera parte del horno el flujo a contracorriente de gases será la forma de calentamiento inicial de la pasta cerámica. Posteriormente a esta zona de entrada, nos encontramos con la ubicación en la parte inferior del canal de quemadores de "alta velocidad", situados de forma lateral, que elevan considerablemente la temperatura ambiental dentro del canal en esa longitud del horno.

Tras esta primera zona de quemadores inferiores, se situarán posteriormente quemadores tanto por encima como por debajo del plano de rodillos, de forma uniforme con respecto a su separación -podría decirse que se colocan en zigzag-. En esta zona tenemos la temperatura más elevada del horno - en torno a los $1200^{\circ} \mathrm{C}$ - que es la utilizada para la cocción propia de la pasta: normalmente dicha pasta necesita unos minutos a una temperatura más ó menos constante para su correcta cocción.

Tras la cocción de la pasta, necesitamos enfriarla hasta su salida a una temperatura adecuada (nunca más de $200^{\circ} \mathrm{C}$ ), controlando así todas las transformaciones físico-químicas que puedan producirse en el proceso de enfriamiento. Para dicho proceso, primero se construye una primera zona de enfriamiento rápido compuesta de diversos tubos verticales que insuflan aire frío directamente sobre la pasta. De esta forma se consigue una reducción considerable de la temperatura que ha alcanzado la pasta en la última zona de cocción. Posteriormente a esta zona, la pasta cerámica debe enfriarse de forma muy lenta, porque el contenido de cuarzo de las arcillas sufren una transformación alotrópica con variación considerable en el volumen de las mismas -dicho proceso ocurre alrededor de los $570^{\circ} \mathrm{C}$-. Tras enfriar de forma controlada esta zona, se vuelven a introducir tubos verticales

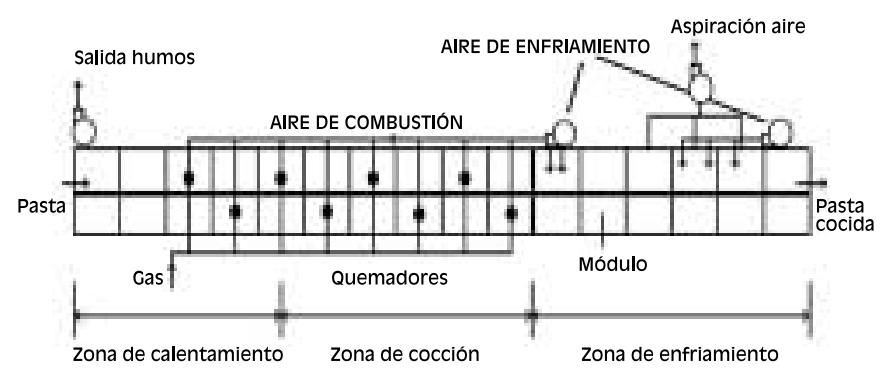

Figura 1. Esquema general de un horno monoestrato.

para enfriar más rápidamente la pasta hasta la temperatura de salida del horno de la misma, en torno a los $150^{\circ} \mathrm{C}$.

La figura 1 resume la configuración básica de este tipo de hornos monoestrato. El canal del horno se encuentra convenientemente aislado del exterior para reducir así las pérdidas de calor del ambiente interior que tenemos. Por último, es reseñable la existencia de ventiladores necesarios para recircular la gran cantidad de flujos de aire y humos que necesita este horno. Se cuenta con un aspirador de humos, que recoge todo el flujo de los gases de combustión que generan los quemadores; y un aspirador de los caudales de aire de enfriamiento -tanto rápido como final-, situado en la parte final del horno. Asímismo, el horno posee un ventilador para insuflar el aire de combustión que van a necesitar los quemadores; otro ventilador montado en la misma sujeción para lanzar los chorros de aire frío a la pasta recién cocida; y finalmente un ventilador para el aire de enfriamiento rápido postrero. El aire de combustión que llega a los quemadores es precalentado a través de un intercambiador aire-aire, dicho aire se calienta con el aire interior de la zona de enfriamiento forzado.

\section{MODELO MATEMÁTICO}

La complejidad de modelado de este horno nos implica la utilización del modelo denominado "modelo de horno largo" ó "Stirred vessel cascade", en el cual el horno se divide en zonas a lo largo de toda su longitud; dichas zonas se considerarán isotermas. La utilización de este modelo implica entre otras estas simplificaciones:

* Uniformidad de temperaturas en todas las superficies del canal interior del horno: Paredes interiores, rodillos y pasta cerámica, para cada división zonal.

* Condiciones isotérmicas del aire ó mezcla de humos en cada zona en que se divide el horno.

* La pasta cerámica se considera una "placa uniforme" que provoca una separación perfecta entre el canal superior ó inferior del horno.

* Mezcla perfecta de gases en el canal en cada zona, teniendo en cuenta que puede haber chorros de enfriamiento forzado y chorros de humos de los quemadores.

* Proceso estacionario: Este tipo de hornos trabajan en contínuo, introduciendo generalmente el mismo tipo de pasta, y por lo tanto a lo largo de tiempo mantienen constantes los mismos valores de temperatura y de presiones.

La construcción modular de este tipo de hornos, y la existencia de los quemadores situados en el centro de cada módulo, hacen necesario para la modelización hacer la 
partición zonal coincidente con dichos módulos prefabricados.

La utilización de quemadores de "alta velocidad", es decir, quemadores con la salida de gases de combustión a velocidades considerables debido a la existencia de un inyector, simplifica la resolución matemática de parte del fenómeno de transmisión de calor por radiación en el horno, ya que dentro del mismo no tenemos llama visible. La transmisión de calor por convección es sólo destacable en la zona de enfriamiento del horno, con la existencia de los chorros de enfriamiento forzado; aunque en la zona de enfriamiento forzado inicial las temperaturas en los módulos son lo suficientemente elevadas para que la transmisión de calor por radiación sea también comparable a la de convección. Por último, el fenómeno de transmisión de calor por conducción sólo nos aparece a la hora de calcular las pérdidas de calor a través de los aislamientos del horno; y en el paso de calor entre la pasta y los rodillos, la modelización del intercambiador de calor aire-aire ó las perdidas de calor a través de los rodillos.

La resolución del modelo del horno es, en esencia, la resolución de un complejo sistema de ecuaciones; dicho sistema es el Balance de Energía de cada módulo del horno. Por lo tanto, se establecerá para cada zona un sistema de ecuaciones no lineales; cada ecuación será el balance de energía de los materiales ó gases existentes en cada módulo (recordar que son considerados a temperatura uniforme). Las incógnitas de dicho sistema son, respectivamente, el salto de temperaturas que experimenta la pasta cerámica, la temperatura de los rodillos, la temperatura de las paredes interiores (tanto superiores como inferiores), y por último la temperatura de la mezcla de humos y/ó aire que pueda circular por el canal -superior e inferior- de cada zona, que cierran el balance de energía total de cada zona.

En la figura 2 se esquematizan las variables a calcular, y el funcionamiento del horno en un módulo (en su canal superior) cualquiera del mismo. Debe tenerse en cuenta que dicho sistema de ecuaciones no lineales para cada módulo está acoplado con los módulos adyacentes, es decir, las temperaturas de cada módulo están relacionadas con las temperaturas con sus módulos vecinos. Esta consideración hace que la resolución del sistema sea mucho más compleja y lenta, repercutiendo en la duración de la resolución del sistema total.

El balance de energía de una zona ó modulo i cualesquiera de los que componen el horno es el siguiente (remitirse a la nomenclatura del final):

$\mathrm{F}_{\mathrm{c}, i}=\mathrm{Q}_{\mathrm{c}, \mathrm{u}}^{\mathrm{rd}}+\mathrm{Q}_{\mathrm{c}, \mathrm{d}}^{\mathrm{rd}}+\mathrm{Q}_{\mathrm{c}, \mathrm{u}}^{\mathrm{cv}}+\mathrm{Q}_{\mathrm{c}, \mathrm{d}}^{\mathrm{cv}}-\mathrm{Q}_{\mathrm{c}-\mathrm{r}}^{\mathrm{cd}}-\mathrm{Q}^{\mathrm{rc}}=0$.

$\mathrm{F}_{\mathrm{r}, \mathrm{i}}=\mathrm{Q}_{\mathrm{r}}^{\mathrm{rd}}+\mathrm{Q}_{\mathrm{r}}^{\mathrm{cv}}+\mathrm{Q}_{\mathrm{c}-\mathrm{r} .}^{\mathrm{cd}}-\mathrm{Q}_{\mathrm{r}}^{\mathrm{al}}=0$.

$\mathrm{F}_{\mathrm{pu}, i}=\mathrm{Q}_{\mathrm{pu}}^{\mathrm{rd}}+\mathrm{Q}_{\mathrm{pu}}^{\mathrm{cv}}-\mathrm{Q}_{\mathrm{pu}}^{\mathrm{cd}}=0$.

$\mathrm{F}_{\mathrm{pd}, i}=\mathrm{Q}_{\mathrm{pd}}^{\mathrm{rd}}+\mathrm{Q}_{\mathrm{pd}}^{\mathrm{cv}}-\mathrm{Q}_{\mathrm{pd}}^{\mathrm{cd}}=0$.

$\mathrm{F}_{h u, i}=\mathrm{Q}_{\mathrm{hu}}^{\mathrm{cb}}+\mathrm{m}_{\mathrm{hu}}^{\mathrm{e}} \mathrm{H}_{\mathrm{hu}}^{\mathrm{e}}-\mathrm{Q}_{\mathrm{hu}}^{\mathrm{rd}}-\mathrm{Q}_{\mathrm{hu}}^{\mathrm{cv}}-\mathrm{m}_{\mathrm{hu}}^{\mathrm{s}} \mathrm{H}_{\mathrm{hu}}^{\mathrm{s}}=0$.

$\mathrm{F}_{\mathrm{hd}, i}=\mathrm{Q}_{\mathrm{hd}}^{\mathrm{cb}}+\mathrm{m}_{\mathrm{hd}}^{\mathrm{e}} \mathrm{H}_{\mathrm{hd}}^{\mathrm{e}}-\mathrm{Q}_{\mathrm{hd}}^{\mathrm{rd}}-\mathrm{Q}_{\mathrm{hd}}^{\mathrm{cv}}-\mathrm{m}_{\mathrm{hd}}^{\mathrm{s}} \mathrm{H}_{\mathrm{hd}}^{\mathrm{s}}=0$.

De dichas ecuaciones, el término radiativo asociado es el más costoso de calcular. Dada la geometría del horno, se escoge el método zonal (1) para calcular el intercambio de calor por radiación a cada superficie ó zona de gas de cada módulo del horno, ya que dicho modelo considera zonas isotermas de gas y superficie. El balance de energía radiante

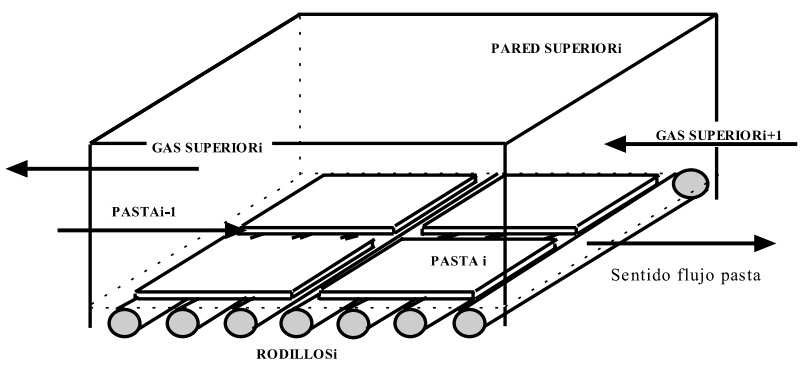

Figura 2. Elementos de una zona superior del horno monoestrato a modelizar.

para una superficie se puede poner en términos de áreas de intercambio dirigidas:

$$
\dot{Q}_{i}=\sum_{j=1}^{m} \overrightarrow{S_{j} S_{i}} E_{j}+\sum_{j=1}^{l} \overrightarrow{G_{j} S_{i}} E_{g, j}-\left[\sum_{j=1}^{m} \overleftarrow{S_{j} S_{i}}+\sum_{j=1}^{l} \overleftarrow{G_{j} S_{i}}\right] E_{i}
$$

y para un volumen

$$
\dot{Q}_{i}=\sum_{j=1}^{l} \overrightarrow{G_{j} G_{i}} E_{j}+\sum_{j=1}^{m} \overrightarrow{S_{j} G_{i}} E_{j}-\left[\sum_{j=1}^{l} \overleftarrow{G_{j} G_{i}}+\sum_{j=1}^{m} \overleftarrow{S_{j} G_{i}}\right] E_{g, i}
$$

Las áreas de intercambio dirigidas se obtienen de la forma:

$$
\begin{aligned}
& \overrightarrow{S_{i} S_{j}}=\sum_{n=1}^{N} a_{n}\left(T_{i}\right)\left(\overline{S_{i} S_{j}}\right)_{K=K n} \\
& \overleftarrow{S_{i} S_{j}}=\sum_{n=1}^{N} a_{n}\left(T_{j}\right)\left(\overline{S_{i} S_{j}}\right)_{K=K n} \\
& {\overrightarrow{G_{i} S_{j}}}_{j}=\sum_{n=1}^{N} a_{n}\left(T_{g, i}\right)\left(\overline{G_{i} S_{j}}\right)_{K=K n} \\
& \overleftarrow{G_{i} S_{j}}=\sum_{n=1}^{N} a_{n}\left(T_{j}\right)\left(\overline{G_{i} S_{j}}\right)_{K=K n} \\
& \overrightarrow{G_{i} G_{j}}=\sum_{n=1}^{N} a_{n}\left(T_{g, j}\right)\left(\overline{G_{i} G_{j}}\right)_{K=K n} \\
& \overleftarrow{G_{i}} \overleftarrow{G}_{j}=\sum_{n=1}^{N} a_{n}\left(T_{g, j}\right)\left(\overline{G_{i} G_{j}}\right)_{K=K n}
\end{aligned}
$$

Los coeficientes de peso para cada paso son:

$$
\begin{aligned}
& \mathrm{a}_{\mathrm{n}}\left(\mathrm{T}_{\mathrm{g}, \mathrm{i}}\right)=\mathrm{b}_{1, \mathrm{n}}+\mathrm{b}_{2, \mathrm{n}} \mathrm{T}_{\mathrm{g}, \mathrm{i}} \\
& \text { ó }
\end{aligned}
$$

$$
\mathrm{a}_{\mathrm{n}}\left(\mathrm{T}_{\mathrm{i}}\right)=\mathrm{b}_{1, \mathrm{n}}+\mathrm{b}_{2, \mathrm{n}} \mathrm{T}_{\mathrm{i}}
$$

dependiendo del modelo de gas, es decir, una suma de componentes grises que se elija. En nuestro caso, como el gas natural produce en su combustión una cantidad despreciable de hollín, se puede coger un modelo 3 componentes grises (2), uno de ellos considerado como gas claro, sin consideran componente de hollín.

A su vez, las matrices de intercambio total anteriormente mencionadas se obtienen a partir de las matrices de intercambio directo (3):

$$
\begin{aligned}
& \overline{\text { SS }}=\varepsilon A I \cdot R \cdot \overline{s S} \cdot \varepsilon I \\
& \overline{\text { SG }}=\varepsilon A I \cdot R \cdot \overline{s g} \\
& \overline{\text { GG }}=\overline{\text { gs. }} \cdot \rho I \cdot R \cdot \overline{\text { sg }}+\overline{\text { gg }}
\end{aligned}
$$


donde

$$
\mathbf{R}=\left[\begin{array}{ll}
\mathbf{A} \mathbf{I}-\overline{\mathbf{s s}} . & \mathbf{I}
\end{array}\right]^{1}
$$

Las matrices de intercambio directo dependen de factores geométricos y de las propiedades espectrales el gas y de las superficies adyacentes. De forma genérica se calculan de la forma (4):

$$
\begin{aligned}
& \overline{\mathrm{s}_{\mathrm{i}} \mathrm{s}_{\mathrm{j}}}=\frac{\dot{\mathrm{Q}}_{i \rightarrow \mathrm{j}}}{\mathrm{E}_{\mathrm{i}}}=\iint_{A \mathrm{~A} A \mathrm{j}} \frac{\cos \vartheta_{\mathrm{i}} \cos \vartheta_{\mathrm{j}} \tau(\mathrm{r})}{\pi \mathrm{r}^{2}} \mathrm{dA}_{\mathrm{j}} \mathrm{dA}_{\mathrm{i}} \\
& \overline{\mathrm{g}_{\mathrm{i}} \mathrm{s}_{\mathrm{j}}}=\frac{\dot{\mathrm{Q}}_{\mathrm{i} \rightarrow \mathrm{j}}}{\mathrm{E}_{\mathrm{g}, \mathrm{i}}}=\iint_{\mathrm{A} \mathrm{j} \mathrm{V}_{\mathrm{i}}} \frac{\mathrm{K}_{\mathrm{i}} \cos \vartheta_{\mathrm{j}} \tau(\mathrm{r}) \mathrm{dV}_{\mathrm{i}} \mathrm{dA}_{\mathrm{j}}}{\pi \mathrm{r}^{2}} \\
& \overline{\mathrm{g}_{\mathrm{i}} \mathrm{g}_{\mathrm{j}}}=\frac{\dot{\mathrm{Q}}_{\mathrm{i} \rightarrow \mathrm{j}}}{\mathrm{E}_{\mathrm{g}, \mathrm{i}}}=\iint_{\mathrm{V}_{\mathrm{j}} \mathrm{Vi}} \frac{\mathrm{K}_{\mathrm{i}} \mathrm{K}_{\mathrm{j}} \tau(\mathrm{r})}{\pi \mathrm{r}^{2}} \mathrm{dV}_{\mathrm{i}} \mathrm{dV}_{\mathrm{j}}
\end{aligned}
$$

El término de convección incluye todos los fenómenos de transmisión de calor entre una superficie y un campo fluido. La aplicación de los coeficientes de convección ó transferencia global de calor $h$ va a simplificar el cálculo de este modo de transferencia de calor. Lógicamente, con la complicada geometría del horno monocapa (sobre todo la existencia del banco de rodillos de movimentación), ha sido necesario adecuar las correlaciones experimentales existentes para los distintos modos de intercambio de calor entre diferentes superficies y flujos. Por otra parte, la existencia de diferentes flujos de aire y gases en direcciones perpendiculares -existe el flujo horizontal que circula por los canales, y los chorros de los quemadores ó las toberas de enfriamiento perpendiculares al flujo principal del canal-, obliga a la aplicación de teorías sobre la transmisión de calor de flujos transversales. Si $N u_{1}$ y $\mathrm{Nu}_{2}$ son los números de Nusselt de los intercambios de calor por convección de esos flujos individuales, el número de Nusselt global en esa zona es de la forma:

$$
\overline{N u}_{T}=\left(\overline{N u}_{1}^{3}+\overline{N u}_{2}^{3}\right)^{1 / 3},
$$

donde

$$
\overline{N u}=\frac{h K}{L}
$$

siendo h: Coeficiente de convección $\left(\mathrm{W} / \mathrm{m}^{2} \mathrm{~K}\right), \mathrm{K}$ : Conductividad térmica del fluido $(\mathrm{W} / \mathrm{m} \mathrm{K})$, y L: Dimensión característica del recinto de intercambio $(\mathrm{m})$. (Recordar que $\mathrm{q}=\mathrm{h} \cdot \mathrm{A} \cdot(\mathrm{Ts}-\mathrm{T} \infty)$, siendo A el área de la superficie de contacto, y Ts, To las temperaturas de la superficie de contacto y del fluido). Las diferentes correlaciones utilizadas para calcular los flujos de calor convectivos en función de la disposición fluido superficie son las siguientes (5),(6):

$$
\begin{aligned}
& \mathrm{Nu}=0.0296 \operatorname{Re}_{\mathrm{x}}^{4 / 5} \operatorname{Pr}^{1 / 3}, \text { para los chorros de los quemadores } \\
& \mathrm{Nu}=\frac{(\mathrm{f} / 8)(\operatorname{Re}-1000) \mathrm{Pr}}{1+12.7(\mathrm{f} / 8)^{1 / 2}\left(\operatorname{Pr}^{2 / 3}-1\right)}, \text { para el flujo de aire ó gases en el canal } \\
& \mathrm{Nu}=\mathrm{C}_{2} 1.13 \mathrm{C}_{1} \operatorname{Re}_{\mathrm{D}, \max }^{\mathrm{m}} \operatorname{Pr}^{1 / 3}, \text { para el flujo cruzado de los rodillos } \\
& \mathrm{Nu}=1.16 \mathrm{D}_{\text {eq }}^{0.6} \operatorname{Re}^{0.6} \operatorname{Pr}^{1 / 3}\left(\frac{\mu}{\mu_{\mathrm{s}}}\right)^{0.14}, \text { para el flujo paralelo a los rodillos }
\end{aligned}
$$

$\mathrm{Nu}=\left\{0.60+\frac{0.387 \mathrm{Ra}_{\mathrm{D}}^{1 / 6}}{\left[1+(0.559 / \mathrm{Pr})^{9 / 16}\right]^{-/ 27}}\right\}^{2}$, para la convección natural de los rodillos (30)
$\mathrm{Nu}=0.54 \mathrm{Ra}_{\mathrm{L}}^{1 / 4}$ ó
$\mathrm{Nu}=0.27 \mathrm{Ra}_{\mathrm{L}}^{1 / 4}$, para convección natural en placas horizontales
$\mathrm{Nu}=\left\{0.825+\frac{0.387 \mathrm{Ra}_{\mathrm{L}}^{1 / 6}}{\left[1+(0.492 / \mathrm{Pr})^{9 / 16}\right]^{/ 27}}\right\}^{2}$, conv. natural en p. verticales (32)

$\mathrm{Nu}=\mathrm{F}_{2}(\mathrm{Re}) \mathrm{G}\left(\frac{\mathrm{H}}{\mathrm{D}}, \mathrm{A}_{\mathrm{r}}\right) \mathrm{K}\left(\mathrm{A}_{\mathrm{r}}, \frac{\mathrm{H}}{\mathrm{D}}\right) \operatorname{Pr}^{0.42}$, para chorros de enfriamiento

con $\mathrm{F}_{2}=0.5 \mathrm{Re}^{2 / 3} ; \quad \mathrm{G}=2 \mathrm{~A}_{\mathrm{r}}^{1 / 2} \frac{1-2.2 \mathrm{~A}_{\mathrm{r}}^{1 / 2}}{1+0.2\left(\frac{\mathrm{H}}{\mathrm{D}}\right) \mathrm{A}_{\mathrm{r}}^{1 / 2}}, \mathrm{~K}=\left[1+\left(\frac{\mathrm{H} / \mathrm{D}}{0.6 / \mathrm{A}_{\mathrm{r}}^{1 / 2}}\right)^{6}\right]^{-0.05}, \quad \mathrm{~A}_{\mathrm{r}}=\frac{\pi \mathrm{D}^{2}}{4 \mathrm{~S}^{2}}$.

Los términos conductivos del balance de energía pueden contener las pérdidas de calor del horno a través de una cualquiera de sus paredes en un proceso iterativo, ya que nuestro dato es la temperatura de la cara interior del horno, y dicha temperatura variará en cada iteración que realicemos en la ejecución del programa; además la resistencia de contacto entre la cerámica y los rodillos (7) y las pérdidas radiales a través de los rodillos también son mecanismos de transmisión por conducción.

En el balance de energía correspondiente a las zonas donde tenemos quemadores, aparece el flujo de calor que aportan los quemadores de alta velocidad. Es necesario modelizar la combustión de dichos quemadores para incorporar al balance de energía dicho flujo de calor que entra en cada módulo, así como la composición de los humos generados por la combustión completa del gas natural, con el exceso de aire diferente en cada caso. Los quemadores utilizados, contienen una cámara de combustión donde se produce la misma. La salida de los gases de combustión se efectúa a través de un inyector, de reducido diámetro, que provoca una evacuación rápida de los productos de la combustión realizada. El elevado índice de aire con el que se está produciendo dicha combustión provoca que no se perciba llama visible en el canal del horno, es decir, lugar de salida de los productos de combustión. La cantidad total de productos de combustión $\mathrm{q}_{\text {tot }}$ por $\mathrm{m}^{3}$ de gas es la suma de los productos formados por la combustión estequiométrica del gas $\left(\mathrm{q}_{\mathrm{st}}\right)$ y el exceso $(\mathrm{n}-1)$ de aire de combustión que no forma parte en la reacción de combustión -recordar que es una combustión completa con exceso de aire-:

$$
\mathrm{q}_{\mathrm{tot}}=\mathrm{q}_{\mathrm{st}}+(\mathrm{n}-1) \mathrm{a}
$$

La formulación para el cálculo de las diversas componentes de los productos es la siguiente:

$$
\begin{aligned}
& \mathrm{q}_{\mathrm{CO} 2}=\left(\sum_{\mathrm{i}=1}^{5} \mathrm{n}_{\mathrm{i}} \cdot \mathrm{CO}_{2, \mathrm{i}}+\mathrm{n}_{\mathrm{CO} 2, \mathrm{~g}}+\mathrm{n} \cdot \mathrm{n}_{\mathrm{CO} 2, \mathrm{a}} \cdot \mathrm{a}\right) \frac{\mathrm{z}_{\mathrm{CO} 2}}{\mathrm{z}_{\mathrm{g}} 100} \quad\left(\mathrm{~m}^{3} \mathrm{CO}_{2} / \mathrm{m}^{3} \text { gas }\right) . \\
& \mathrm{q}_{\mathrm{H} 2 \mathrm{O}}=\left(\sum_{\mathrm{i}=1}^{5} \mathrm{n}_{\mathrm{i}} \cdot \mathrm{H}_{2} \mathrm{O}_{\mathrm{i}}+\mathrm{n} \cdot \mathrm{n}_{\mathrm{H} 2 \mathrm{O}, \mathrm{a}} \cdot \mathrm{a}\right) \frac{\mathrm{z}_{\mathrm{H} 2 \mathrm{O}}}{\mathrm{z}_{\mathrm{g}} 100}\left(\mathrm{~m}^{3} \mathrm{H}_{2} \mathrm{O} / \mathrm{m}^{3} \text { gas }\right) . \\
& \mathrm{q}_{\mathrm{N} 2}=\left(\mathrm{n}_{\mathrm{N} 2, \mathrm{~g}}+\mathrm{n} \cdot \mathrm{n}_{\mathrm{N} 2, \mathrm{a}} \cdot \text { a }\right) \frac{\mathrm{z}_{\mathrm{N} 2}}{\mathrm{z}_{\mathrm{g}} 100}\left(\mathrm{~m}^{3} \mathrm{~N}_{2} / \mathrm{m}^{3} \text { gas }\right) .
\end{aligned}
$$


$\mathrm{q}_{\mathrm{Ar}}=\left(\mathrm{n} \cdot \mathrm{n}_{\mathrm{Ar}, \mathrm{a}} \cdot \mathrm{a}\right) \frac{\mathrm{z}_{\mathrm{Ar}}}{\mathrm{z}_{\mathrm{g}} 100} \quad\left(\mathrm{~m}^{3} \mathrm{Ar} / \mathrm{m}^{3} \mathrm{gas}\right)$.

$\mathrm{q}_{\mathrm{O} 2}=(\mathrm{n}-1) \cdot \mathrm{n}_{\mathrm{O} 2, \mathrm{a}} \cdot \mathrm{a} \cdot \frac{\mathrm{z}_{\mathrm{O} 2}}{\mathrm{z}_{\mathrm{g}} 100} \quad\left(\mathrm{~m}^{3} \mathrm{O}_{2} / \mathrm{m}^{3}\right.$ gas $)$.

$\mathrm{q}_{\mathrm{st}}=\sum_{\mathrm{i}=1}^{5} \mathrm{q}_{\mathrm{i}}(\mathrm{n}=1) \quad\left(\mathrm{m}^{3} / \mathrm{m}^{3}\right.$ gas $)$

$\mathrm{q}_{\text {tot }}=\sum_{\mathrm{i}=1}^{5} \mathrm{q}_{\mathrm{i}}(\mathrm{n} \neq 1) \quad\left(\mathrm{m}^{3} / \mathrm{m}^{3}\right.$ gas $)$.

El valor del aire estequiométrico a necesario para la combustión de un gas natural se calcula con la siguiente fórmula (8):

$$
\mathrm{a}=\frac{z_{O 2}\left[\left(\sum_{i=1}^{5} n_{i} \cdot O_{2, i}\right)-n_{O 2, g}\right]}{z_{g} \cdot x_{O 2, g}}
$$

La regulación de los quemadores del horno se efectúa a través de un índice característico para cada quemador. Dicho índice será un dato a introducir exteriormente por el usuario, y guarda una estrecha relación con el consumo de g.n que se va a gastar en el horno. De esta forma tenemos que:

$$
\begin{aligned}
& \mathrm{C}_{\text {stg }}=(\text { Aircomb } / \text { Nqval }) / \mathrm{a} \\
& \text { Cons }_{\text {gas }}=\mathrm{I}_{\text {util }} \cdot \mathrm{C}_{\text {stg }} \\
& \mathrm{n}=(\text { Aircomb } / \mathrm{Nqval}) /\left(\mathrm{a} \cdot \text { Cons }_{\text {gas }}\right. \text { ), }
\end{aligned}
$$

Por lo tanto, con la utilización de este índice, con unos márgenes adecuados de funcionamiento que no permitan un índice de la combustión muy elevado $(n>20)$, podemos calcular el flujo de calor que aportan los quemadores al horno, considerando también la energía que aporta el aire previamente calentado en el intercambiador situado en la zona de enfriamiento rápido inicial:

$\mathrm{Q}_{\text {quemador }}=\left(\mathrm{C}_{\text {stg }} \cdot 1000 \cdot \mathrm{PCI}_{\text {gas }}\right) / 3600+($ Aircomb $/ \mathrm{Nqval}) \cdot \mathrm{H}\left(\mathrm{T}_{\mathrm{a}}\right.$

En los balances de energía de cada módulo, aparece de forma implícita el balance de masa, tanto en el canal inferior como en el superior del horno. La división de la pasta en dos canales independientes, no supone para el cálculo del balance de masa una separación de los canales; es decir, para el cálculo del balance de masa en cualquiera de los canales se considera que por los mismos circula la misma cantidad de humos de combustión ó aire de enfriamiento. Además, hay que recordar la simplificación de la suposición de mezcla perfecta de los humos que circulan por el canal con los chorros de enfriamiento ó la salida de humos de los quemadores. Esta mezcla perfecta también se utilizará a la hora de calcular las presiones parciales de los componentes gaseosos existentes en las diferentes atmósferas del horno, presiones muy importantes para determinar el comportamiento final de las pastas tras su cocción.

Toda la cantidad de calor que llega a la pasta, a través de sus diferentes modos de transmisión, se consume en la elevación de la temperatura de dicha pasta, y en la realización de diferentes reacciones físicas ó químicas que se puedan producir en el proceso de cocción.

$$
\sum Q p=\dot{m} c p\left(T p_{i+1}-T p_{i}\right)+\text { Qreaccion }
$$

donde $\dot{m}$ es el flujo másico $(\mathrm{kg} / \mathrm{s})$ de la pasta cerámica que recorre el horno, y $c p$ el calor específico de la pasta cerámica ( $\mathrm{J} / \mathrm{kg} \mathrm{K})$, variable en función de la temperatura de la pasta en cada módulo. $\mathrm{Tp}_{\mathrm{i}+1}, \mathrm{Tp}_{\mathrm{i}}$, son las temperaturas de la pasta a la entrada y salida de un módulo cualquiera, y Qreacción ${ }_{\mathrm{i}}$ es el calor de reacción necesario en ese módulo, que depende de la temperatura y composición de la pasta que estemos cociendo. Las reacciones más significativas, dignas de tener en consideración son, para las componentes mineralógicas, las siguientes:

Carbonatos: Sufren la calcinación alrededor de los $870^{\circ} \mathrm{C}$, con un gasto de $425 \mathrm{kcal} / \mathrm{kg}$.

Caolín: Sufre el proceso de deshidroxilación a los $500^{\circ} \mathrm{C}$, con un gasto $19.4 \mathrm{kcal} / \mathrm{kg}$.

Illita: Sufre el mismo proceso que el caolín, pero a los $550^{\circ}$ C, con un consumo de $300 \mathrm{kcal} / \mathrm{kg}$.

Cuarzo: La transición del $\alpha$-cuarzo al $\beta$-cuarzo consume a los $573^{\circ} \mathrm{C}$ un total de $4.8 \mathrm{kcal} / \mathrm{kg}$.

\section{MÉTODO SOLUCIÓN}

El método escogido es el Newton Raphson generalizado; este método es idéntico al Newton-Raphson sólo que se sustituye la matriz jacobiana del sistema por una matriz diagonal, de la siguiente manera:

$$
F^{\prime}(\mathbf{x})=\left(\frac{\partial f_{i}}{\partial x_{i}}\right)
$$

y de la misma forma, sustituye ésta por sus coeficientes incrementales

$$
\frac{\partial f_{i}}{\partial x_{i}}\left(\mathbf{x}^{(i)}\right) \approx \frac{f_{i}\left(\mathbf{x}^{(i)}+e_{i} h\right)-f_{i}\left(\mathbf{x}^{(i)}\right)}{h} .
$$

$\mathrm{Al}$ aproximar esta matriz, el sistema a resolver en cada iteración es inmediato, y esto disminuye mucho el tiempo de cálculo, no sólo por la resolución del sistema sino porque se disminuye el número de cálculos del jacobiano al calcularse sólo los términos de la diagonal.

Este método es el que se ha aplicado a la resolución del balance de energía del horno, por su menor costo computacional. A pesar de que se observa una convergencia más lenta que el caso del Newton (necesita un número mayor de iteraciones para converger), queda compensado con el hecho de que las iteraciones son mucho más rápidas. Además, la utilización de la matriz diagonal en vez de la matriz jacobiana implica un cierto desacoplamiento entre las diferentes superficies y masas de gas de cada módulo, que ayuda a la convergencia del sistema (la ejecución del programa por Newton solía siempre llegar a la divergencia del sistema de ecuaciones).

\section{RESULTADOS Y CONCLUSIONES}

A través del programa de simulación se puede realizar un estudio de optimización paramétrica de diversas opciones que el programa puede variar, entre ellos tenemos la composición del gas y de las pastas cerámicas, número y dimensión de los módulos, rodillos, quemadores, caudales de aire de entrada y condiciones ambientales. 
El efecto de la calidad de un gas natural en este tipo de hornos no es significativo de cara al resultado final obtenido: las temperaturas finales de los componentes de cada módulo varían tan sólo una décima de grado en el caso más desfavorable para diferentes gases naturales introducidos. En cambio, la cantidad de gas consumida sí que varia considerablemente de unos a otros gases, para compensar sus diferentes poderes caloríficos (puede llegar en torno al 20\% de variación entre los gases más ricos y más pobres energéticamente de los suministrados en España). La atmósfera resultante del horno tampoco varía sustancialmente de unos a otros gases estudiados: las diferencias mayores que se obtienen son de un $0.2 \%$ de $\mathrm{O}_{2}$ en la zona del cortafuegos (lógicamente será la zona donde más note el cambio de un gas de suministro), dicha variación no es problemática si los porcentajes de oxígeno en el canal se mantienen por encima de unos márgenes de seguridad. Se puede decir que la variación del aire estequiométrico que supone un cambio de g.n. se ve compensada por la variación -en sentido contrario- del poder calorífico del nuevo gas, manteniendo fijo el aire de combustión.

La composición, formato y ciclo de cocción de la pasta a utilizar es un dato a introducir en el programa, por lo tanto se podrá estudiar el efecto de variación de cualquiera de estos parámetros. Los caudales de aire de entrada, tanto los del aire de combustión como los del aire de enfriamiento, son parámetros fundamentales en la evolución de la simulación en el horno. El caudal del aire primario a los quemadores es el responsable del fenómeno de combustión en el horno. Con un razonamiento similar al del aire de combustión se denota la importancia de la elección adecuada de los caudales de aire de enfriamiento, en función de la carga cerámica introducida (tanto peso como composición de la misma). Será insuflado el aire necesario para que las piezas cocidas salgan a $150^{\circ} \mathrm{C}$, asegurándonos de un enfriamiento muy lento alrededor de los $570^{\circ} \mathrm{C}$ (transformación alotrópica del cuarzo). Los caudales de aire deben estar en relación a las dimensiones del horno que simulemos: si el horno es muy ancho y de canal muy largo, los caudales de combustión y de enfriamiento deberán ser también elevados.

El número de módulos del horno se podrá variar a voluntad del usuario, dentro de unos márgenes lógicos. De esta forma, se puede estudiar el efecto del aumento del número de módulos de alguna de las zonas típicas del horno: prehorno, horno, diferentes zonas de enfriamiento, etc., en las curvas de temperatura finales de la pasta cerámica. También se podría estudiar el ahorro económico considerando diferentes tamaños del mismo, conjugando el coste de los materiales y la producción obtenible en cada caso. Las zonas que pueden variarse, que luego se mostrarán en el manual de usuario del programa, son las siguientes:

(1) Zona de módulos situada entre los dos tubos de la chimenea de aspiración de humos.

(2) Zona de módulos entre la zona de quemadores de prehorno y el segundo tubo de la chimenea de aspiración de humos.

(3) Zona de prehorno: quemadores inferiores.

(4) Zona de horno: quemadores superiores e inferiores.

(5) Zona de transformación alotrópica del cuarzo.

(6) Zona de enfriamiento final (número de módulos cerrados y en uso).

Las dimensiones del módulo del horno se podrán variar a nuestra voluntad, dentro de unos límites razonables que permitan que la modelización del problema no esté afectada seriamente. Con dichas variaciones podremos estudiar, por ejemplo, la altura del canal del horno para un aprovechamiento energético óptimo del mismo. La anchura del canal es un factor ya más estudiado por los fabricantes de hornos, pero por ejemplo la longitud de cada módulo también es un parámetro importante, ya que influye en otras consideraciones tales como la disposición de los quemadores en ese nuevo módulo.

Se permiten analizar también los dos tipos de rodillos más utilizados en este tipo de hornos. Respecto a los quemadores, además de poder introducir el número deseado, con su índice de operación correspondiente, se podrá variar el diámetro de la boquilla de salida de los inyectores de cada quemador. De forma lógica, se puede intuir que un diámetro de boquilla mayor provoca una velocidad menor del chorro de gases de combustión que salen por cada módulo, y por lo tanto el horno ve desfavorecida la transmisión de calor hacia la pasta. Dicho efecto se puede apreciar claramente cambiando dentro de unos límites dicho diámetro.

Finalmente, dentro del programa se puede variar tanto la temperatura exterior de la nave como la humedad relativa de la misma, para ver qué efecto tienen dichas variaciones sobre los valores finales de temperatura de la pasta a cocer. Normalmente, dicho efecto no será muy apreciable, ya que las variaciones de dichos valores van a ser poco comparables con los valores de temperatura en los que nos movemos.

Además de los parámetros puramente de diseño del horno monocapa, el horno puede estudiar el efecto de otros valores que han sido introducidos por defecto en el mismo: así como ejemplo se podría estudiar la variación de las propiedades superficiales y térmicas de los materiales utilizados en la construcción del mismo: conductividades, emisividades, etc. Otros parámetros podrían ser la disposición y el número de toberas en la zona de enfriamiento, separación entre dichos tubos, etc.

Los resultados globales de consumo de gas y consumo específico por $\mathrm{kg}$. de pasta a cocer están en consonancia con los datos aportados con la industria castellonense, para idénticas curvas de cada tipo de pasta y simuladas en el horno monocapa (ver tabla I).

TABLA 1. COMPARACIÓN DE CONSUMO PARA UN FORMATO DE UNA PASTA CARACTERÍSTICA DEL PROGRAMA CON DATOS REALES (MONOCOCCIÓN DE PAVIMENTO ROJO).

\begin{tabular}{|l|c|c|}
\hline & $\begin{array}{c}\text { Consumo gas } \\
\left(\mathrm{Nm}^{3} / \mathrm{h}\right)\end{array}$ & $\begin{array}{c}\text { Consumo específico } \\
(\mathrm{kcal} / \mathrm{kg})\end{array}$ \\
\hline Industria cerámica & $140-160$ & $550-650$ \\
\hline SHOMOR & 148.1 & 586.3 \\
\hline
\end{tabular}

\section{BIBLIOGRAFÍA}

1. H. C. Hottel, A. F. Sarofim. "Radiative Transfer". Ed. McGraw-Hill Company, New York, 1962.

2. H. C. Hottel, E. S. Cohen. "Radiant Heat Exchange in a Gas-Filled Enclosure: Allowance for Nonuniformity of Gas Temperature". A.I.Ch.E. Journal 4 [1] 3-14 (1958).

3. J. M. Rhine, R. J. Tucker. "Modelling of Gas Fired Furnaces and Boilers". British Gas Company, London, 1991.

4. R. V. Dunkle. "Geometric Mean Beam Lengths for Radiant Heat-Transfer Calculations". Journal of Heat Transfer 88, 75-80 (1964) 
5. H. Martin. "Heat and mass transfer between Impinging Gas Jets and Solid Surfaces". Advances in Heat Transfer 13, 1-60 (1977).

6. J. M. Marín, C. Monné. "Apuntes de transmisión de calor". Universidad de Zaragoza, 1995

7. A. Bejan. "Theory of Rolling Contact Heat Transfer". Journal of Heat Transfer 111, 257-263 (1989).

8. T. M. Geersen. "Physical Properties of Natural Gases". N. V. Nederlandse Gasunie, 1988.

\section{NOMENCLATURA}

F: Función vectorial del balance de energía de cada módulo del horno.

Q: Flujo de calor (kW)

$\mathrm{m}$ : Flujo másico de aire ó gases $(\mathrm{kg} / \mathrm{s})$.

H: Entalpía de gases de combustión ó aire.

E: Poder emisivo $\left(\mathrm{W} / \mathrm{m}^{2}\right)$.

$a_{i}, b_{i}$ : Coeficientes de peso para ponderación de un gas real como suma de gases grises.

T: Temperatura (K)

$\varepsilon$ : Emisividad de superficie ó gas.

$\rho$ : Reflectividad de superficie.

A: Matriz diagonal con las áreas de las superficies del modelo.

I: Matriz unitaria diagonal.

$\mathrm{K}_{\mathrm{i}}$ : Coeficiente de absorción de un gas $\left(\mathrm{m}^{-1} \mathrm{~atm}^{-1}\right)$.

$\theta$ : Angulo de incidencia de una superficie y su vector normal.

$\tau$ : Transmitividad de superficie ó gas.

Nu: Número de Nusselt.

h: Coeficiente de convección $\left(\mathrm{W} / \mathrm{m}^{2} \mathrm{~K}\right)$.

$\mathrm{K}$ : Conductividad de un material ó fluido $(\mathrm{W} / \mathrm{m} \mathrm{K}$ ).

Re: Número de Reynolds.

Pr: Número de Prandtl.

Ra: Número de Raileigh

: Factor de fricción.
$\mathrm{C}_{1}, \mathrm{C}_{2}$ : Constantes para la correlación de flujo en cruzado en rodillos.

D: Diámetro (m).

$\mu$ : Viscosidad de un fluido.

$\mathrm{H}$ : Altura de canal (m).

a: Aire estequiométrico.

$\mathrm{S}$ : Superficie $\left(\mathrm{m}^{2}\right)$

$\mathrm{n}$ : Indice de aire de la combustión.

$\mathrm{n}$ : Concentración del componente i (\%mol).

z: Factor de compresibilidad de un gas.

m: caudal másico $(\mathrm{kg} / \mathrm{s})$

Cons $_{\text {gas }}$ : Consumo de gas por quemador $\left(\mathrm{m}^{3}\right)$

$\mathrm{C}_{\mathrm{stg}}$ : Consumo estequiométrico gas $\left(\mathrm{m}^{3}\right)$

Aircomb: Caudal aire combustion $\left(\mathrm{Nm}^{3} / \mathrm{h}\right)$

Nqval: Número quemadores utilizados

$\mathrm{I}_{\text {util }}$; Indice utilización de cada quemador.

$\mathrm{Q}_{\text {quemador }}:$ Calor aportado por cada quemador a su módulo $(\mathrm{kW})$.

$\mathrm{PCI}_{\text {gas }}$ : Poder calorífico inferior del gas natural $(\mathrm{kJ} / \mathrm{m} 3)$.

q: volumen específico $\left(\mathrm{m}^{3} / \mathrm{m}^{3}\right)$

\section{Subíndices:}

$i$, j: Superficie ó zona de volumen i, j.

hu, pu, hd, pd, c, r: Humos y paredes arriba y abajo en el canal, pasta y rodillos. $\mathrm{g}$, a: Gas, aire.

$\mathrm{x}, \mathrm{L}$ : Longitudes de placa.

$\mathrm{n}$ : Indice de componente en sumatorios.

st: Estequiométrico.

tot: Total

útil: Utilización

stg: Estequiométrico del gas.

\section{Superíndices:}

rd, cv, cd, r, al: Transmisión de calor por radiación, convección, conducción, rodillos ó aletas respectivamente.

e, s: Entrada y salida de gases a un módulo.

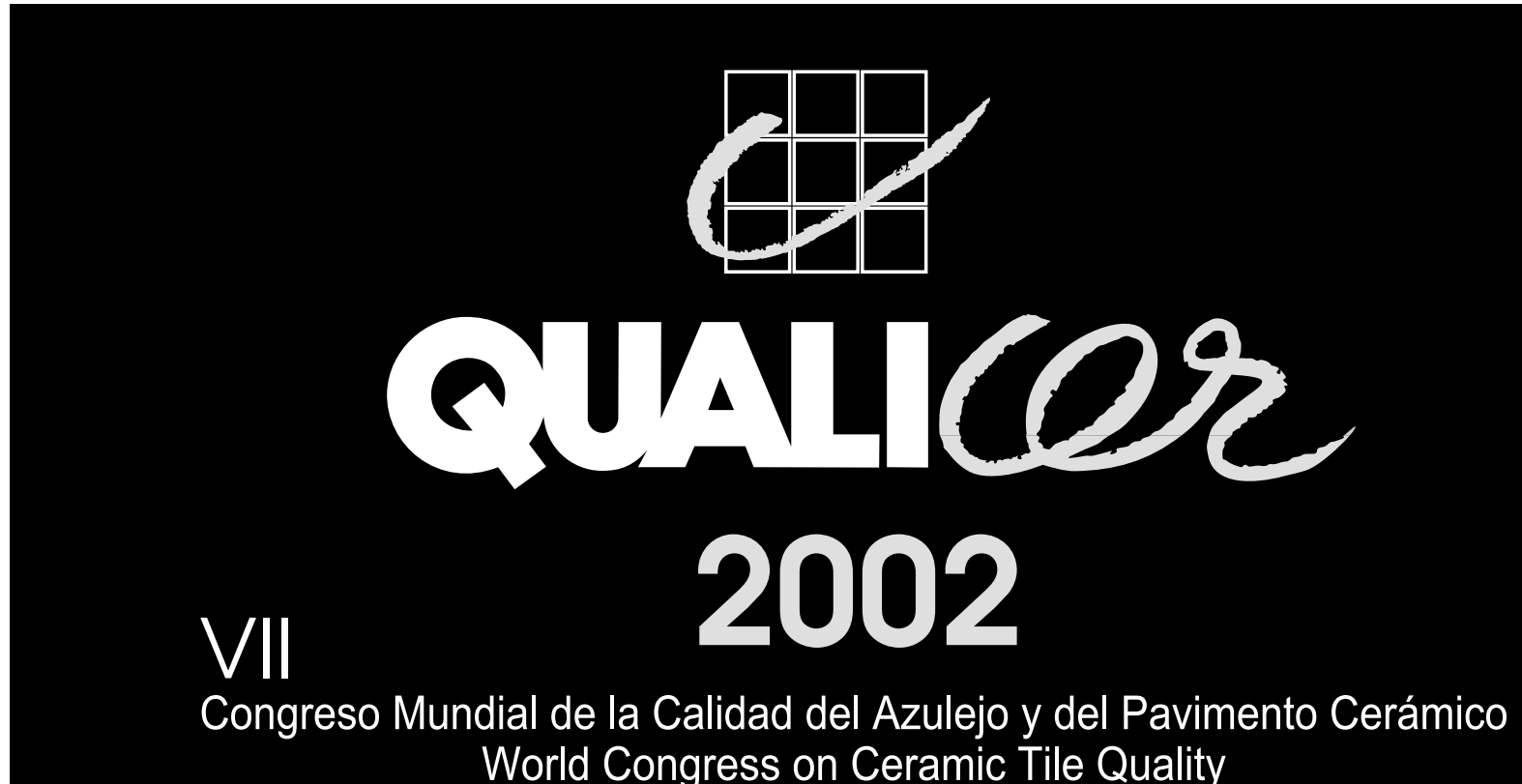

World Congress on Ceramic Tile Quality

\section{CastellónsPAIN}

